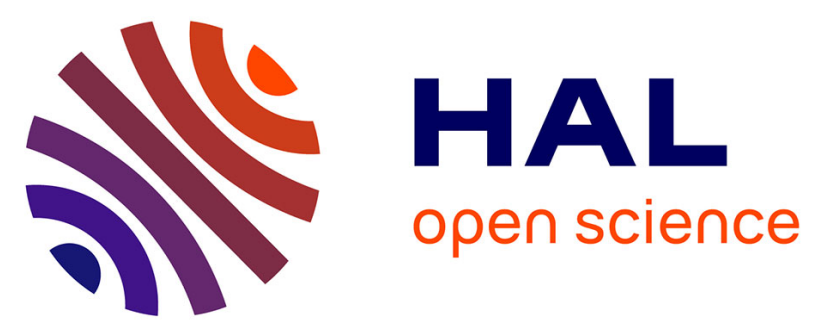

\title{
Application d'une nouvelle méthodologie d'analyse de risques a priori dans un service de radiothérapie
}

Sylvie Thellier, Gianfranco Brusadin, Eric Deutsch, Simon Corbin

\section{To cite this version:}

Sylvie Thellier, Gianfranco Brusadin, Eric Deutsch, Simon Corbin. Application d'une nouvelle méthodologie d'analyse de risques a priori dans un service de radiothérapie. 32ième congrès de la Société Française de Radiothérapie Oncologique, SFRO 2021, Oct 2021, La défense, France. pp.642644, 10.1016/j.canrad.2021.06.007 . hal-03508914

\section{HAL Id: hal-03508914 https://hal.science/hal-03508914}

Submitted on 3 Jan 2022

HAL is a multi-disciplinary open access archive for the deposit and dissemination of scientific research documents, whether they are published or not. The documents may come from teaching and research institutions in France or abroad, or from public or private research centers.
L'archive ouverte pluridisciplinaire HAL, est destinée au dépôt et à la diffusion de documents scientifiques de niveau recherche, publiés ou non, émanant des établissements d'enseignement et de recherche français ou étrangers, des laboratoires publics ou privés.

\section{(ㅇ)(1) $\$$}

Distributed under a Creative Commons Attribution - NonCommercial - NoDerivatives $\mid 4.0$ 
Application d'une nouvelle méthodologie d'analyse de risques a priori dans un service de radiothérapie

Application of a new methodology of a priori risk analysis in a radiotherapy department

\author{
Sylvie Thellier ${ }^{1}$, Gianfranco Brusadin ${ }^{2}$, Éric Deutsch ${ }^{2} \&$ Simon Corbin $^{2}$ \\ ${ }^{1}$ Institut de Radioprotection et de Sûreté Nucléaire (Fontenay-aux-Roses) \\ ${ }^{2}$ Institut Gustave Roussy (Villejuif)
}

\title{
1. Introduction
}

Depuis 2017, l'IRSN développe une nouvelle méthode d'analyse des risques a priori [3]. Cette méthode est mobilisée en radiothérapie pour identifier et gérer les risques encourus par les patients pouvant survenir dans des situations de travail quotidiennes, dont l'organisation réelle est en écart avec l'organisation prescrite. Cette méthode des Espaces de Partage et d'Exploration de la Complexité du Travail (EPECT) a été expérimentée pour la première fois dans son intégralité en 2020 dans le département de radiothérapie de l'Institut Gustave Roussy (IGR). Les résultats de cette expérimentation ont permis de compléter les principes méthodologiques établis en 2017 [3]. Cet article présente l'organisation des trois types d'espaces de discussion nécessaires à l'analyse des risques et la synthèse des résultats d'application.

\section{Description des principes méthodologiques des EPECT}

Différentes méthodes d'analyse des risques a priori - issues des sciences de l'ingénierie - ont été développées à partir de principes méthodologiques similaires [3]. La méthode AMDEC est l'une d'entre elles et elle est classiquement mobilisée dans le domaine médical en France pour identifier et gérer les risques encourus par les patients. Mais les spécificités du domaine médical malmènent les principes méthodologiques de l'AMDEC [2] [6]. Face aux difficultés d'application de l'AMDEC, l'IRSN a développé une nouvelle méthode [3].

La méthode EPECT se distingue des méthodes classiques d'analyse de risques, au moins à quatre niveaux. Premièrement, l'objet de la réflexion n'est pas le même puisque la méthode EPECT analyse les modes de réussite d'une équipe soignante plutôt que ses modes de défaillance. Deuxièmement, la nature des risques identifiés est différente. L'EPECT permet de caractériser les dimensions organisationnelles et contextuelles des situations potentiellement risquées pour les patients. Cette méthode ne cherche pas à identifier des risques théoriques et génériques. Troisièmement, la temporalité de la méthode EPECT n'est pas la même que celle des analyses de risque de type AMDEC. L'EPECT engage l'établissement de santé dans une démarche d'amélioration continue en organisant deux à trois analyses de risques par an, comme dans le cadre des Comités de Retour d'Expérience (CREX). Quatrièmement, le nombre et le type d'espace de discussion (EDD) à mobiliser sont différents de ceux mobilisés dans la méthode AMDEC tels qu'ils sont décrits dans le guide de l'ASN [1]. En effet, la méthode EPECT ne se limite pas à la succession d'un seul type d'EDD [1].

Trois Espaces de discussion différents sont organisés et reliés entre eux par des temps de réflexion et de synthèse (cf. Figure $n^{\circ} 1$ ). 


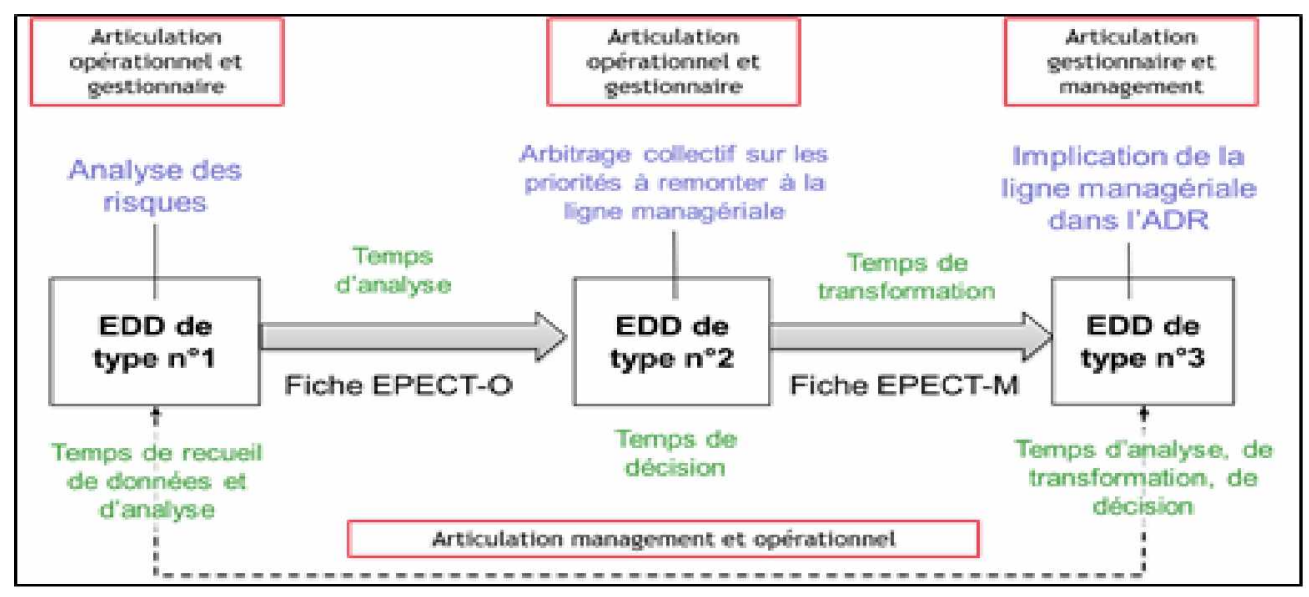

Figure 1 : Représentation schématique des phases de l'analyse des risques de type EPECT

Le premier EDD est dédié à la réalisation de l'analyse des risques (EDD $\left.{ }^{\circ} 1\right)$. La description détaillée des étapes de l'EDD n ${ }^{\circ} 1$ est publiée par ailleurs [3] [4] [5]. Le deuxième EDD permet de décider collectivement les résultats qui seront remontés à la ligne managériale. Pour ce faire, les participants de l'EDD $\mathbf{n}^{\circ} 1$ remettent en discussion dans 1'EDD $\mathrm{n}^{\circ} 2$ certains éléments de l'analyse des risques opérationnelle, notamment les leviers de sécurisation des soins pour choisir ceux qu'ils souhaitent mettre en discussion à un niveau managérial. Le troisième EDD implique directement la ligne managériale dans l'analyse des risques en présentant les résultats principaux des analyses des risques opérationnelles. Pour favoriser les échanges, l'animateur de la restitution élabore un jeu de cinq à six diapositives pour chaque analyse présentant :

- le contexte de la situation de travail étudiée et les éléments mettant en tension l'équipe soignante ;

- les différences entre l'organisation prévue et l'organisation réelle ;

- les situations risquées pour les patients ;

- les leviers d'amélioration de la sécurité des soins identifiés par le niveau opérationnel ;

- les réflexions que l'animateur des EDD (responsable opérationnel qualité/sécurité des soins) propose d'organiser (groupes de travail, groupe d'amélioration continue, décisions à prendre, remontée des besoins).

Cet EDD n³ permet à la ligne managériale de s’approprier les résultats de l'analyse des risques opérationnelle, d'enrichir son regard sur les situations de travail réelles et potentiellement risquées, de la (ou les) compléter et d'intégrer les résultats des analyses des risques aux projets en cours ou à venir du service de radiothérapie.

La synthèse des résultats des analyses conduites à l'IGR est présentée dans le tableau récapitulatif $\mathrm{n}^{\circ} 1$.

\begin{tabular}{|c|c|c|}
\hline $\begin{array}{l}\text { Thématique } \\
\text { retenue }\end{array}$ & $\begin{array}{c}\text { Principaux modes de réussite et / ou situation } \\
\text { risqués }\end{array}$ & $\begin{array}{l}\text { Actions retenues par la ligne } \\
\text { managériale }\end{array}$ \\
\hline $\begin{array}{l}\text { Replanification } \\
\text { dosimétrique d'un } \\
\text { traitement }\end{array}$ & $\begin{array}{l}\text { Manque d'homogénéité dans le signalement d'une } \\
\text { modification anatomique. } \\
\text { Manque d'homogénéité dans la définition et dans } \\
\text { la communication du niveau d'urgence de prise en } \\
\text { charge de la replanification. } \\
\text { Mise en tension des équipes et désorganisation } \\
\text { des activités. }\end{array}$ & $\begin{array}{l}\text { Mettre en place un groupe de travail } \\
\text { afin de : } \\
\text { - définir la pertinence et le niveau } \\
\text { d'urgence/priorité des } \\
\text { replanifications } \\
\text { - harmoniser les pratiques }\end{array}$ \\
\hline
\end{tabular}




\begin{tabular}{|c|c|c|}
\hline & $\begin{array}{l}\text { Absence de définition du gain clinique pour } \\
\text { justifier la décision de replanification avec ou non } \\
\text { l'interruption du traitement en cours. } \\
\text { Risque de confusion entre l'ancienne et la } \\
\text { nouvelle prescription. }\end{array}$ & $\begin{array}{l}\text { Déployer un workflow de tâches } \\
\text { spécifique pour les replanifications } \\
\text { dans le SIRT. }\end{array}$ \\
\hline $\begin{array}{l}\text { Indisponibilité des } \\
\text { médecins pour } \\
\text { valider les images au } \\
\text { début du traitement } \\
\text { (MEP) }\end{array}$ & $\begin{array}{l}\text { Temps d'attente élevé du patient sur la table de } \\
\text { traitement et risque de non détection de } \\
\text { mouvements éventuels. } \\
\text { Retard au poste de traitement pour les patients } \\
\text { suivants avec une mise en tension de l'équipe. } \\
\text { Précipitation dans la validation de l'imagerie par } \\
\text { le médecin. }\end{array}$ & $\begin{array}{l}\text { Mettre en place la validation de la } \\
\text { MEP par le médecin en temps différé } \\
\text { et à distance. } \\
\text { Déployer les pratiques avancées des } \\
\text { manipulateurs en électroradiologie (via } \\
\text { un protocole de coopération). }\end{array}$ \\
\hline $\begin{array}{l}\text { Processus } \\
\text { d'envoi/réception } \\
\text { des « dossiers } \\
\text { techniques } \\
\text { antérieurs » (DTA) } \\
\text { de et vers l'extérieur }\end{array}$ & $\begin{array}{l}\text { Risque de confusion et absence de définition } \\
\text { claire des règles sur la récupération et l'envoi des } \\
\text { DTA ce qui entraine : } \\
\text { - Risque de perte d'information par rapport à un } \\
\text { antécédent de radiothérapie } \\
\text { - Récupération du dossier en retard par rapport à } \\
\text { la date de début de traitement prévue avec une } \\
\text { mise en tension des équipes et des reports de } \\
\text { traitements } \\
\text { - Interruption de la continuité de la prise en } \\
\text { charge des dossiers (organisation basée sur les } \\
\text { personnes) } \\
\text { - Retard dans l'envoi du dossier technique } \\
\text { demandé par un centre extérieur. } \\
\text { Méconnaissance et absence de la définition des } \\
\text { solutions informatiques à utiliser pour l'échange } \\
\text { des fichiers. }\end{array}$ & $\begin{array}{l}\text { Formaliser le processus d'envoi et de } \\
\text { réception du DTA } \\
\text { Assurer l'accessibilité et le partage des } \\
\text { informations sur le réseau } \\
\text { informatique. } \\
\text { Rendre les médecins autonomes sur la } \\
\text { réalisation de la fusion de l'imagerie } \\
\text { du DTA }\end{array}$ \\
\hline
\end{tabular}

Tableau 1 : Synthèse des résultats des analyses des risques de type EPECT.

\section{Conclusion}

L'originalité de la méthode EPECT est d'ancrer l'analyse de risques a priori dans des situations de travail quotidiennes et d'y impliquer la ligne managériale.

Le temps d'engagement des équipes est comparable voire inférieur à un temps d'analyse des risques classique (4 EDD de 2 heures pour 3 scénarios analysés + 1 EDD managérial). En revanche, le temps consacré à l'analyse en dehors des EDD est plus important (6 journées d'analyse et de synthèse par un binôme composé du gestionnaire des risques et d'un autre professionnel).

Le rapport coût/bénéfices peut être considéré comme avantageux puisque la méthode permet d'accéder à une richesse d'informations sur le fonctionnement quotidien de l'organisation des unités de radiothérapie, difficilement accessible autrement. Les informations ainsi recueillies permettent d'envisager des actions pertinentes tant au niveau technique qu'organisationnel afin de sécuriser les soins et de les articuler avec des 
projets qui relèvent d'évolutions technologiques, organisationnelles et de l'amélioration de la qualité de la prise en charge des patients. 


\section{Références}

[1] ASN 2008. Guide d'auto-évaluation des risques encourus par les patients en radiothérapie externe Guide ASN $\mathrm{n}^{\circ} 4$ (ASN/DIS/2008-186).

[2] COOK, R., \& RASMUSSEN, J. (2005). "Going solid": a model of system dynamics and consequences for patient safety. Quality \& Safety in Health Care, 14(2), 130-134.

[3] THELLIER, S. (2017). Approche ergonomique de l'analyse des risques en radiothérapie : de l'analyse des modes de défaillances à la mise en discussion des modes de réussite. Thèse de doctorat en ergonomie. Conservatoire National des Arts et Métiers, Paris, soutenue le 12 décembre 2017, 294 p.

[4] THELliER, S., JEFFroY, F., CUVELIER, L., \& FALZON, P. (2018). L'analyse des risques en radiothérapie : quelle alternative à l'AMDEC ? Lambda Mu 21, 16 - 18 octobre 2018, Reims, France.

[5] THELLIER, S. (2019). Analyse des risques en radiothérapie. Partie 2 : des modes de défaillances aux modes de réussite, un changement de paradigme. Radioprotection 54(1): 21-30. https://doi.org/10.1051/radiopro/2019003

[6] VINCENT, C., \& AMALBERTI, R. (2016). Safer Healthcare - Stratégies for the real world. New York, NY: Springer International Publishing. 